\title{
Novel Dose Dependent Effects of Andrographolide on the Enhancement of Chondrogenesis and Osteogenesis in Human Mesenchymal Stem Cells
}

\author{
Thitianan Kulsirirat ${ }^{1}$, Sittisak Honsawek ${ }^{2}$, Mariko Takeda-Morishita ${ }^{3}$, Nuttanan \\ Sinchaipanid $^{1}$, Jiraporn Leanpolchareanchai ${ }^{1}$, and Korbtham Sathirakul ${ }^{1}$ \\ ${ }^{1}$ Mahidol University Faculty of Pharmacy \\ ${ }^{2}$ Chulalongkorn University Faculty of Medicine \\ ${ }^{3}$ Kobe Gakuin University Faculty of Pharmaceutical Sciences Graduate School of \\ Pharmaceutical Sciences
}

July 2, 2020

\begin{abstract}
Background and Purpose: Andrographolide (AG) is a labdane diterpenoid herb, which is isolated from the leaves of Andrographis paniculate, and widely used for its potential medical properties. However, there are no reports on the effects of AG on the human suprapatellar fat pad of osteoarthritis patients. In the present study, our goal was to evaluate the innovative effects of AG on viability and Tri-lineage differentiation of human mesenchymal stem cells from suprapatellar fat pad tissues. Experimental approach: The effects of Andrographolide on viability and differentiation of human primary mesenchymal stem cells obtained from human suprapatellar fat pad tissues were evaluated by staining assay. Moreover, the effects on molecular expression were quantitatively measured by mRNA expression in real time PCR. Key Results: The results revealed that AG had no cytotoxic effects when the concentration was less than $12.5 \mu \mathrm{g} / \mathrm{mL}$. Interestingly, AG had significantly enhanced, dose dependent, osteogenesis and chondrogenesis as evidenced by a significantly intensified stain for Alizarin Red S, Toluidine Blue and Alcian Blue. Moreover, AG can upregulate the expression of genes related to osteogenic and chondrogenic differentiation, including Runx2, OPN, Sox9, and Aggrecan in mesenchymal stem cells from human suprapatellar fat pad tissues. In contrast, AG suppressed adipogenic differentiation as evidenced by significantly diminished Oil Red O staining and expression levels for adipogenic-specific genes for PPAR- $\gamma 2$ and LPL. Conclusions and Implications: These findings confirm that AG can specifically enhance osteogenesis and chondrogenesis of mesenchymal stem cells from human suprapatellar fat pad tissues. It has potential as a therapeutic agent derived from natural sources for regenerative medicine.
\end{abstract}

Novel Dose Dependent Effects of Andrographolide on the Enhancement of Chondrogenesis and Osteogenesis in HumanSuprapatellar Fat Pad Derived Mesenchymal Stem Cells

Thitianan Kulsirirat ${ }^{1}$, Sittisak Honsawek ${ }^{2}$, Mariko Takeda-Morishita ${ }^{3}$, Nuttanan Sinchaipanid ${ }^{4}$, Jiraporn Leanpolchareanchai ${ }^{1}$, Korbtham Sathirakul ${ }^{*}$

${ }^{1}$ Department of Pharmacy, Faculty of Pharmacy, Mahidol University, Bangkok, Thailand 10210

${ }^{2}$ Osteoarthritis and Musculoskeleton Research Unit, Department of Biochemistry, Faculty of Medicine, Chulalongkorn University, Bangkok, Thailand 10330

${ }^{3}$ Laboratory of Drug Delivery Systems, Faculty of Pharmaceutical Sciences, Kobe Gakuin University, Kobe, Hyogo, Japan 650-8586 
${ }^{4}$ Department of Manufacturing Pharmacy, Faculty of Pharmacy, Mahidol University, Bangkok, Thailand 10400

*Corresponding author. Korbtham Sathirakul, Department of Pharmacy, Faculty of Pharmacy, Mahidol University, Bangkok, Thailand

E-mail address: pyksk2001@yahoo.com.sg

Keywords: Mesenchymal stem cells, Suprapatellar fat pad, Andrographolide, Osteoarthritis, Regenerative medicine

ABSTRACT:

Background and Purpose: Andrographolide (AG) is a labdane diterpenoid herb, which is isolated from the leaves of Andrographis paniculate, and widely used for its potential medical properties. However, there are no reports on the effects of AG on the human suprapatellar fat pad of osteoarthritis patients. In the present study, our goal was to evaluate the innovative effects of AG on viability and Tri-lineage differentiation of human mesenchymal stem cells from suprapatellar fat pad tissues.

Experimental approach: The effects of Andrographolide on viability and differentiation of human primary mesenchymal stem cells obtained from human suprapatellar fat pad tissues were evaluated by staining assay. Moreover, the effects on molecular expression were quantitatively measured by mRNA expression in real time PCR.

Key Results: The results revealed that AG had no cytotoxic effects when the concentration was less than $12.5 \mu \mathrm{g} / \mathrm{mL}$. Interestingly, AG had significantly enhanced, dose dependent, osteogenesis and chondrogenesis as evidenced by a significantly intensified stain for Alizarin Red S, Toluidine Blue and Alcian Blue. Moreover, AG can upregulate the expression of genes related to osteogenic and chondrogenic differentiation, including Runx2 , OPN ,Sox9, and Aggrecan in mesenchymal stem cells from human suprapatellar fat pad tissues. In contrast, AG suppressed adipogenic differentiation as evidenced by significantly diminished Oil Red $O$ staining and expression levels for adipogenic-specific genes for ППAP- 22 and $L P L$.

Conclusions and Implications: These findings confirm that AG can specifically enhance osteogenesis and chondrogenesis of mesenchymal stem cells from human suprapatellar fat pad tissues. It has potential as a therapeutic agent derived from natural sources for regenerative medicine.

Abbreviations: AG, andrographolide; OA, osteoarthritis; IL-1 $\beta$, interleukin-1 $\beta \cdot$ MMPs, matrix metalloproteinases; BMPs, bone morphogenetic proteins; IGF1, insulin growth factor 1; TKA, total knee arthroplasty; SPFP, Suprapatellar fat pad; MSCs, mesenchymal stem cells; PBS, phosphate buffer solution; DMEM-HG, Dulbecco's Modified Eagle Medium-high Glucose; FBS, fetal bovine serum; SPFP-MSCs, suprapatellar fat pad derived mesenchymal stem cells; ISCT, International Society for Cellular Therapy; DMSO, dimethyl sulfoxide; OD, optical density; IBMX, 3-isobutyl-methyl-xantine; TGF- $\beta 3$, transforming growth factor- $\beta 3$; ITS, insulin-transferrin-selenium; H\&E, Haematoxylin \& Eosin; $\mathrm{C}_{\mathrm{T}}$, cycle threshold; GAPDH, glyceraldehyde3-phosphate dehydrogenase; OM, osteogenic induction medium; Runx2, Runt-related transcription factor 2; OPN , Osteopontin; AM, adipogenic induction medium; ППAP- $\gamma 2$, Peroxisome proliferator-activated receptor gamma 2; LPL, lipoprotein lipase; CM, chondrogenic medium; Sox 9 ,SRY -related transcription factor 9; NF- $x \mathrm{~B}$, nuclear factor-kappaB; ERK, extracellular-signal-regulated kinase

\section{What is already known}

* The early stage of the OA is a significant increase in bone turnover and re-modelling.

* Andrographolide has a similar size to estrogen and may contain estrogen like activities.

\section{What this study adds}

* Andrographolide could promote chondrogenic and osteogenic without adipogenic differentiation of SPFPMSCs in dose dependent manner. 
* Andrographolide is associated with regulation of osteocyte and chondrocyte specific markers and its antiadipocyte effect.

\section{What is the clinical significance}

* Andrographolide can be an innovative compound for cartilage and bone regeneration in OA treatment.

* An appropriate Andrographolide formulation could be developed for future use in human regenerative medicine.

\section{INTRODUCTION}

Osteoarthritis (OA) is one of the most common debilitating diseases encountered globally. The clinical symptoms of OA are pain and functional impairment that includes joint stiffness and dysfunction. It affects 30 million people in the U.S.(Lawrence et al., 2008). In $80 \%$ of patients with OA have some degree of movement impairment. This leads to diminished performance in the workplace, and $25 \%$ of patients cannot perform the main activities of their daily life, which often leads to social isolation and depression(Kean, Kean, \& Buchanan, 2004). Nevertheless, in spite of decades of drug research and development, no disease-modifying drug for osteoarthritis has been approved for use in humans(Gerwin, Hops, \& Lucke, 2006). Such a drug could slow progression by reducing the rate of cartilage degeneration. The current standard of care focuses on pain relief only after symptoms are present, by the use of acetaminophen, NSAIDs COX2 inhibitors and corticosteroids.

One of the hallmarks of $\mathrm{OA}$ is the pathological structural changes that occur in the subchondral cortical, trabecular bone and subarticular structures. The early stage in the course of the disease is a significant increase in bone turnover and re-modelling (both bone formation and resorption) of the bone cartilage interface, especially in areas underlying damaged cartilage. The trabecular bone, and the cortical subchondral plate thickens and becomes increasingly irregular (Buckland-Wright, 2004). The biomechanical factors, such as localized increased load on the subarticular bone beneath areas of damaged cartilage, and pathobiochemical influences, such as enhanced release of cytokines and tissue growth factors (Haringman, Ludikhuize, \& Tak, 2004; Sweeney \& Firestein, 2004). Key contributors to catabolic processes include, interleukin-1 $\beta$ (IL-1 $\beta$ ) and matrix metalloproteinases (MMPs), growth factors and free radicals, among others(Steinberg \& Zeggini, 2016). Bone morphogenetic proteins (BMPs) and insulin growth factor 1 (IGF1) are endogenous anabolic factors that stimulate cartilage generation and remodelling(Fan et al., 2004; Lajeunesse, 2004; Matsumoto, Gargosky, Iwasaki, \& Rosenfeld, 1996; Sandell \& Aigner, 2001). In addition, health problems of excessive osteoclast formation and bone resorption can cause an imbalance in bone remodeling and thus induce many adult skeletal diseases, including osteoporosis, osteoarthritis, rheumatoid arthritis, multiple myeloma and metastatic cancers, premature menopause and low levels of testosterone and estrogen in men, all potentially leading to changes in cartilage homeostasis.

Andrographolide (AG) is the major active principle isolated from the plant Andrographis paniculate and having a similar size or containing an estrogen-like molecule(Cauley et al., 2008). It is a well-known traditional medicinal plant in India, Thailand, and China. This plant is widely recognized for its therapeutic and biologically active properties. Many studies have focused on andrographolide $\left(\mathrm{C}_{20} \mathrm{H}_{30} \mathrm{O}_{5}\right)$ activity, for examples, anticancer(Khan, Khan, Farooqui, \& Ansari, 2018; Liao et al., 2019), anti-viral(Paemanee, Hitakarun, Wintachai, Roytrakul, \& Smith, 2019; Wintachai et al., 2015), anti-thrombotic(Lu et al., 2012; Mussbacher et al., 2019), hepatoprotective(Chua, 2014; Singha, Roy, \& Dey, 2007), and anti-inflammatory properties(Tan, Liao, Zhou, \& Wong, 2017). Furthermore, AG has been reported to have inhibitory effects on osteoclastogenesis and osteoclast function in vitro and in vivo (Zhai et al., 2014). Due to the lack of cytotoxicity and evidence of the effects of AG on multilineage differentiation in human mesenchymal stem cells, this study's aimed is to investigate the effects of AG on the in-vitro cytotoxicity, and human mesenchymal stem cell differentiation in order to identify potential mechanisms of action.

Stimulating bone and cartilage formation with biologically active pharmaceuticals derived from natural sources is a direction worth investigating for the treatment of homeostasis of bone diseases and/or bone 
remodeling in regenerative medicine field.

\section{MATERIALS AND METHODS}

Andrographolide $\left(\mathrm{C}_{20} \mathrm{H}_{30} \mathrm{O}_{5},>98 \%\right.$ purity) was purchased from Tokyo Chemical Industry Co., Ltd., (Tokyo, Japan). Cultures of human tissue samples were collected from the suprapatellar fat pad tissue as surgical waste from osteoarthritis patients aged over 60 years who underwent total knee arthroplasty (TKA). Informed consent was obtained before surgery. The study protocol was approved by the Institution Review Board (MUDT/PY-IRB 2018/037.1309) at Faculty of Dentistry/Faculty of Pharmacy, Mahidol University, Bangkok, Thailand prior to performing the study.

\subsection{MSCs isolation, expansion and culture}

Suprapatellar fat pad (SPFP) tissues were harvested during TKA with tissues and then used for cell isolation, and for collecting mesenchymal stem cells (MSCs) according to a previously published protocol(Katagiri et al., 2017). In brief, SPFP tissues from resections were rinsed and washed with phosphate buffer solution (PBS) (Gibco, Life Technologies, NY, USA), and then chopped in to small pieces. After that, $0.1 \%$ collagenase type I solution (Gibco, Life Technologies, NY, USA) was added and incubated under warm-water bath $\left(37^{[?]} \mathrm{C}\right)$ for 60 minutes then suspended with Dulbecco's Modified Eagle Medium-high Glucose (DMEM-HG) (Gibco, Life Technologies, NY, USA) containing 10\% fetal bovine serum (FBS) (Gibco, Life Technologies, NY, USA ) and centrifuged at 400x for 10 minutes and the surfactant layer was removed. The resulting pellet was mixed with $15 \mathrm{~mL}$ of complete medium (DMEM-HG $+10 \% \mathrm{FBS}+1 \%$ L-glutamic acid (Gibco, Life Technologies, NY, USA) + 1\% Pen-strep (Gibco, Life Technologies, NY, USA), then passed through a sterile filter/cell strainer (Corning, NY, USA) and finally seeded in T-175 tissue culture flasks (Wuxi NEST Biotechnology, Jiangsu, China) which were placed in a $37^{[?]} \mathrm{C}$ incubator with $5 \% \mathrm{CO}_{2}$. Culture medium was replaced every 3 days. If cells expanded to more than $80 \%$ of the culture flask, then SPFP cells were detached with $0.05 \%$ Trypsin/0.1\% EDTA (Gibco, Life Technologies, NY, USA) and recultured as the first passage with complete medium through $2^{\text {nd }}$ passage. Cell count and time between each passage were recorded. Suprapatellar fat pad derived mesenchymal stem cells ( SPFP-MSCs) in the first passage were trypsinized and divided for the following assays following International Society for Cellular Therapy (ISCT).

\subsection{Cell viability assay}

SPFP-MSCs from passage 1 were seeded into 96 -well plates at a density of $1 \times 10^{4}$ cells/well in triplicate for 24 hours and then the cells were incubated with control blank medium (fresh DMEM) and blank medium with increasing concentrations of AG $(1.56,3.125,6.25,12.5,25,50$ and $100 \mu \mathrm{M})$ for $24 \mathrm{hrs}$. Next, the cells in each well were washed with PBS and replaced by $100 \mu \mathrm{l}$ of $0.5 \mathrm{mg} / \mathrm{ml}$ MTT solution. The cells were then incubated for another 4 hours at $37{ }^{\circ} \mathrm{C}$ under $5 \% \mathrm{CO} 2$, after which the MTT solution in each well was carefully discarded and replaced by $50 \mu \mathrm{l}$ of dimethyl sulfoxide (DMSO) to dissolve formazan crystals. The optical density (OD) was then measured with multimode microplate reader (Infinite M200 PRO, Tecan Group Ltd., Männedorf, Switzerland) at a wavelength of $590 \mathrm{~nm}$. The cell viability was calculated relative to the control using the following formula: (sample group OD - zeroing OD)/ (control group OD - zeroing OD).

\subsection{In vitro Tri-lineage differentiation and histological analysis}

Passage 1 SPFP-MSCs samples from ten knee osteoarthritis patients were subjected to directed differentiation to induce osteogenesis, adipogenesis, and chondrogenesis (Table 1). All directed-differentiation media were freshly prepared in house.

Osteogenesis: SPFP-MSCs were seeded at a cell density of 5,000 cells $/ \mathrm{cm}^{2}$ in osteogenic medium, prepared freshly in house and containing $10 \mathrm{nM}$ dexamethasone, $10 \mathrm{mM} \beta$-glycerophosphate and $50 \mu \gamma / \mu \lambda$ ascorbate-2 phosphate (all Sigma-aldrich, MO, USA) and with 10\% FBS. SPFP-MSC cultures were maintained into AG containing media at various concentrations $(1,5$, and $10 \mu \mathrm{M})$ for 21 days. Medium was changed every 3 days. The extracellular calcium deposits were determined from $100 \%$ ethanol-fixed cell cultures by incubating with 
$0.2 \% \mathrm{w} / \mathrm{v}$ Alizarin Red S solution for 40 minutes at room temperature. Excess dye was removed by several washing steps using water and observed under an inverted light microscope.

Adipogenesis: SPFP-MSCs were seeded at a cell density of 5,000 cells $/ \mathrm{cm}^{2}$ and when the SPFP-MSCs were $>90 \%$ confluent, the growth medium was substituted with in-house, freshly prepared differentiation medium containing $10 \mu \mathrm{g} / \mathrm{mL}$ insulin, $100 \mathrm{nM}$ dexamethasone, $0.45 \mathrm{mM}$ IBMX (3-isobutyl-methyl-xantine), and 50 $\mu \mathrm{g} / \mathrm{mL}$ indomethacin (all from Sigma-Aldrich, MO, USA). The cells were then incubated in AG-containing media at varying concentrations $(1,5$, and $10 \mu \mathrm{M})$ for 21 days. The adipogenic differentiation was evaluated from formalin-fixed cell cultures by incubating with $0.3 \%$ (w/v) Oil Red O (Sigma) solution for 15 minutes at room temperature. Then, the excess staining was washed and removed with water. The stained lipid vacuoles were observed under inverted light microscope.

Chondrogenesis: The SPFP-MSCs were cultured to form a cell pellet in a $15 \mathrm{~mL}$ polypropylene tube (Wuxi NEST Biotechnology, Jiangsu, China). Approximately $2 \times 10^{5}$ cells were cultured in chondrogenic medium with in-house ,freshly prepared medium with the presence of $10 \mathrm{nM}$ dexamethasone (Sigma-Aldrich, MO, USA), $10 \mathrm{ng} / \mathrm{ml}$ transforming growth factor- $\beta 3$ (TGF- $\beta 3$ ) (ProSpec, Rehovot, Israel) and $6.25 \mu \mathrm{g} / \mathrm{ml}$ insulin-transferrin-selenium (ITS supplement) (MP Biomedicals, California, USA) were expanded. The cells were then incubated with andrographolide-containing media at different concentrations $(1,5$, and $10 \mu \mathrm{M})$ for 21 days with medium changes every 3 days. Alcian blue, Toluidine blue, and Haematoxylin \& Eosin (H\&E) were used to detect the presence of enrichment of glycosaminoglycans in cartilage, polysaccharides and morphology, respectively. Before staining, the cell pellet cultures were fixed in formalin then embedded in paraffin and sectioned into 4-5 $\mu \mathrm{m}$ and stained with H\&E, Alcian blue and Toluidine blue. The sections were observed under an inverted light microscope.

\subsection{RNA isolation and real-time PCR analysis}

The differences between the messenger RNA (mRNA) levels of osteogenic, adipogenic and chondrogenic related genes in stem cell differentiation of andrographolide at different concentrations $(1,5$, and $10 \mu \mathrm{M})$ were analyzed using real-time PCR. Total RNA was extracted on days 0 and 21 of each induction period using a FavorPrep Blood/ Cultured Cell Total RNA Mini Kit (FAVORGEN, Ping-Tung, Taiwan) according to the manufacturer's instructions. Real-time PCR was conducted using SensiFAST SYBR ${ }^{\circledR}$ Lo-ROX one step kits. Quantitative mRNA analysis was performed by the Mx 3005p Real Time PCR system (Stratagene, Agilent Technologies Germany GmbH \& Co. KG, Baden-Württemberg, Germany). Specific primer pairs for each gene as shown in Table 2. The expression level of each gene level was calculated by the comparative cycle threshold $\left(\mathrm{C}_{\mathrm{T}}\right)$ method and normalized with that of the housekeeping gene, glyceraldehyde-3-phosphate dehydrogenase (GAPDH). A comparison of mRNA expression in each sample was calculated based on the differences in $\Delta \mathrm{CT}$ of individual samples $(\Delta \Delta \mathrm{CT})$. Graphs show the relative expression levels compared with the control on day 0 .

\subsection{Data and statistical analysis}

Statistical analyses were performed using SPSS version 20.0 (IBM, USA). All Data are expressed as the mean \pm standard deviation (SD). $P$-value of $<0.05$ was considered statistically significant in this study. Analysis of variance was calculated for comparison in demographic data of patients, MSCs immunophenotypes, and gene expression of SPFP-MSCs in each source.

\section{RESULTS}

\subsection{Isolation and culture expansion ofsuprapatellar fat pad derived mesenchymal stem cells}

Cells from SPFP samples were isolated by enzymatic digestion. After cultures had been incubated for 3 days, the isolated cells markedly spread out and adhered to the plastic culture flask. By $4-5$ days, cells exhibited homogenous spindle-shaped, fibroblast-like morphology. As time went on, the cells evinced a high proliferation rapidly and took on a clustered appearance. Cultures reached $80-90 \%$ confluence in $7-10$ days. Following the first sub-culturing, approximately 4-5 days were needed for each passage. Cell morphology remained homogeneous until being terminated (Fig. 1). 


\subsection{Effect of Andrographolide on the cell viability of differentiating suprapatellar fat pad derived mesenchymal stem cells}

To investigate the effect of AG on cell viability, the MTT assay was performed by measuring mitochondrial activity. The result showed that the OD values of SPFP-MSCs were stable following treatment with AG which did not influence cell viability when it was used at concentrations between 1.56 and $12.5 \mu \mathrm{M}$, indicating that cytotoxicity was undetectable when the concentration of AG was less than $12.5 \mu \mathrm{M}$. However, AG demonstrated the ability to decrease cell viability significantly at dosages above $12.5 \mu \mathrm{M}$, suggesting that high concentrations of andrographolide could inhibit cell proliferation of SPFP-MSCs (Fig. 2).

\subsection{In vitro tri-lineage differentiation and histological analysis}

First-passage of cells cultures were subjected to in vitrodifferentiation assays in order to evaluate their mesenchymal multipotent potential. The shape of the cells changed to a spindle-shaped morphology. Cells demonstrated a potential to differentiate to varying degrees as shown by positive osteogenic, adipogenic, and chondrogenic staining.

In osteogenesis differentiation, cells seeded at $5 \times 10^{4}$ cells per well were seeded in 6 well plates in triplicate in culture medium. After 24 hours, confluent cell populations (90\% confluency) from SPFP-MSCs were promoted by treating cells cultures with osteogenic induction medium (OM) containing AG at different concentrations $(1,5$, and $10 \mu \mathrm{M})$ while cells maintained in OM were used as controls. After 3 weeks, cells were stained with Alizarin Red S to confirm the accumulation of calcium (Fig. 3A). Furthermore, their osteogenic potency was confirmed by examining mRNA expression of several osteogenic-specific marker genes including Runt-related transcription factor 2 (Runx2) and Osteopontin (OPN ) (Fig. 4A). OM containing AG10 $\mu \mathrm{M}$ profoundly upregulated the mRNA expression of osteogenic markers compared to control groups of cells.

Using these isolated cells from SPFP, we further determined the adipogenic potential of cells with cultures using adipogenic induction medium (AM), these cells became more elongated and flatter. $5 \times 10^{4}$ cells per well were seeded in 6 well plates in triplicate in expansion medium. After 24 hours, confluent cell populations (90\% confluency) from SPFP were promoted by treating expansion medium cultures with AM containing AG at different concentrations $(1,5$, and $10 \mu \mathrm{M})$. SPFP-MSCs were observed, especially at the sites where lipid droplets became visible and compared with SPFP-MSCs maintained in AM for 3 weeks as control groups. After 3 weeks, lipid droplets were confirmed by Oil Red O staining to visualize accumulated cytoplasmic lipid rich vacuoles (Fig. 3B). According to real-time PCR assay, expression levels of adipogenic-specific genes for Peroxisome proliferator-activated receptor gamma $2(\Pi \Pi A P-\gamma 2)$ and Lipoprotein lipase $(L P L)$ (Fig. 4B), the mRNA expression showed significantly lower in induced groups with AM containing AG at all concentrations $(1,5$, and $10 \mu \mathrm{M})$ than in control groups.

Further analysis made was to evaluate chondrogenic differentiation capacity (pellets) of isolated MSCs from SPFP. Pellets were either introduced to chondrogenic medium (CM) containing AG at different concentrations $(1,5$, and $10 \mu \mathrm{M})$ or CM with $1 \%$ FBS. The cells had grown and formed dense spheroidal pellets (Fig. 3C). After 21 days, the spheroidal pellets were easily processed for staining with H\&E (Fig. 3D), Toluidine blue (metachromatic) (Fig. 3E) and Alcian blue (Fig. 3F). The results showed morphology as demonstrated by H\&E, the presence of glycosaminoglycans as demonstrated by Toluidine blue and the presence of enrichment of sulphated proteoglycans as illustrated by Alcian blue staining. The mRNA expression levels of chondrogenic-specific genes including SRY-related transcription factor 9 (Sox9) and Aggrecan (Fig. 4C) showed significantly higher in the induction pellets containing AG at all concentrations $(1,5$, and $10 \mu \mathrm{M})$ than in the CM as control group.

\section{DISCUSSION}

The number of elderly patients in need of treatment related to bone formation has increased, and they may have a decreased capability of bone remodeling. Therefore, stimulating bone and cartilage formation from natural sources that selectively enhance osteogenesis and chondrogenesis without stimulating adipogenesis 
are desirable.

Andrographis paniculata has long been used in traditional medicine in Asia. In Thailand, this medicinal plant was selected by the Ministry of Public Health as one of the medicinal plants to be included in "The National List of Essential Drugs A.D. 1999" (List of Herbal Medicinal Products(Pholphana et al., 2004)). Several studies have shown that its main bioactive component, AG, has a broad range of beneficial pharmacological effects, such as antiviral, anti-mararial, immunostimulatory, and anti-inflammatory. Moreover, it contains bioactive molecules like estrogen. In the present study, we have significant findings. We have demonstrated that AG did not influence cell viability when it was used at concentrations ranging from 1.56 to $12.5 \mu \mathrm{M}$. This indicates that AG did not have an effect on human mesenchymal stem cells. In addition, we also originally demonstrated for the first time that AG promoted osteogenic and chondrogenic differentiation of SPFP-MSCs and suppressed their adipogenic differentiation.

There are two major modes of osteogenesis, and both involve the transformation of a preexisting mesenchymal tissue into chondroblast, chondrocytes, and osteoblasts. The mesenchymal stem cells differentiate into chondroblasts and chondrocytes and this cartilage is later replaced by bone. The important markers for osteogenic differentiation,Runx2 and $O P N$, where Runx2 is a master regulator in the late stage marker of osteogenesis and $O P N$ is the important intermediate stage marker during the differentiation into mature osteoblasts for osteogenic differentiation followed by matrix maturation and matrix mineralization(Jensen et al., 2011; Lee et al., 2000; Lindfors, Heikkilä, \& Aho, 2008; Long, 2001). Zhai et al. also showed that AG exhibits the inhibitory effects on osteoclastogenesis and osteoclast function in vitro and in vivo through the suppression of nuclear factor-kappaB $(\mathrm{NF}-\varkappa \mathrm{B})$ and extracellular-signal-regulated kinase (ERK) signaling pathways (Zhai et al., 2014). After 21 days of culture with SPFP-MSCs, AG stimulated the expression of Runx2 , OPN and increased the calcium deposition activity in a dose-dependent manner, suggesting that AG could enhance the osteogenic ability of SPFP-MSCs.

In order to investigate whether AG could inhibit adipogenic differentiation of SPFP-MSCs, the adipocytespecific markers including ПП $A P-\gamma 2$ and $L P L$ and also qualitative of adipocytes number (Oil Red O staining) were examined. The $\Pi \Pi A P-\gamma 2$ which has been shown to play an important role in mature adipocyte differentiation as a terminal differentiation marker, lipid storage, insulin sensitization and can be activated by fatty acids(Auwerx, 1999; Xu et al., 1999). LPL is thought to be an early marker of adipogenesis and highly expressed during adipogenic differentiation(Shaughnessy, Smith, Kodukula, Storch, \& Fried, 2000). As shown in this study, after 21 days of culture treatment with AG. AG suppressed both ППIP- 22 and $L P L$ mRNA expression by inhibiting adipocyte cells formation. These results suggest that AG could suppress the adipogenic differentiation both qualitatively and quantitatively.

In this study, we used an in vitro cell pellet culture of chondrogenesis for 21 days derived from SPFPMSCs and found the different concentrations of AG were able to significantly enhance differentiation in a dose-dependent manner by upregulating a number of genes associated with chondrogenesis such as Sox 9 and Aggrecan under chondrogenic plus andrographolide extract conditions(de Crombrugghe et al., 2000). Moreover, different concentrations of AG revealed a significant higher cell growth rate than the control. In addition, qualitative study exhibited increased synthesis of matrix proteoglycans and visible formation of glycosaminoglycan when stained with Toluidine blue and Alcian blue(Chamberlain, Fox, Ashton, \& Middleton, 2007). The resulting pellet was fixed with H\&E stain for the identification of chondrocyte morphology and revealed a significant dark stain in doses dependent of AG. The intensities of the stain were dependent on the dose of AG.

In conclusion, this study is the first to demonstrate that Andrographolide in the concentration where cell viability was more than $90 \%$ could promote cell proliferation coupled. Moreover, Andrographolide could promote osteogenic and chondrogenic differentiation in a dose-dependent manner whereas inhibit their adipogenesis of SPFP-MSCs. Furthermore, Andrographolide is associated with regulation of osteocyte-specific markers including Runx2, OPN and chondrocyte-specific markers including Sox9 and Aggrecanactivity, and its anti- adipogenic effect is associated with blocking $\Pi \Pi A P-\gamma 2$ and $L P L$ signaling. Our data also strongly suggested that Andrographolide could be developed and expected for the possibility to translate the results 
to human clinical practice as a treatment for regenerative medicine for cartilage and bone regeneration.

\section{ACKNOWLEDGEMENTS}

This research was financially supported by Thailand Research Fund (TRF) through the Royal Golden Jubilee (RGJ) Ph.D. Program (Grant No. PHD/0056/2559), Thailand.

\section{AUTHOR CONTRIBUTIONS}

Korbtham Sathirakul and Sittisak Honsawek participated in conception or design of the work. Thitianan Kulsirirat conducted experiments and data acquisition. Mariko Takeda-Morishita, Nuttanan Sinchaipanid, and Jiraporn Leanpolchareanchai contributed reagents or analytic tools. Korbtham Sathirakul and Thitianan Kulsirirat analysed and interpreted data. Korbtham Sathirakul and Thitianan Kulsirirat wrote the manuscript.

\section{CONFLICT OF INTEREST}

The authors declare no conflicts of interest.

\section{DECLARATION OF TRANSPARENCY AND SCIENTIFIC RIGOUR}

This Declaration acknowledges that this paper adheres to the principles for transparent reporting and scientific rigour of preclinical research as stated in the BJP guidelines for Design \& Analysis, and as recommended by funding agencies, publishers and other organisations engaged with supporting research.

\section{ORCID}

Korbtham Sathirakul:https://orcid.org/0000-0003-1276-9266

\section{REFERENCES}

Auwerx, J. (1999). PPAR $\gamma$, the ultimate thrifty gene.Diabetologia , 42 (9), 1033-1049. https://doi.org/10.1007/s001250051268

Buckland-Wright, C. (2004). Subchondral bone changes in hand and knee osteoarthritis detected by radiography. Osteoarthritis and Cartilage, 12 Suppl A, S10-9. https://doi.org/10.1016/j.joca.2003.09.007

Cauley, J. A., Wampler, N. S., Barnhart, J. M., Wu, L., Allison, M., Chen, Z., .. Jackson, R. D. (2008). Incidence of fractures compared to cardiovascular disease and breast cancer: the Women's Health Initiative Observational Study. Osteoporosis International : A Journal Established as Result of Cooperation between the European Foundation for Osteoporosis and the National Osteoporosis Foundation of the USA , 19 (12), 1717-1723. https://doi.org/10.1007/s00198-008-0634-y

Chamberlain, G., Fox, J., Ashton, B., \& Middleton, J. (2007). Concise review: mesenchymal stem cells: their phenotype, differentiation capacity, immunological features, and potential for homing. Stem Cells (Dayton, Ohio) , 25 (11), 2739-2749. https://doi.org/10.1634/stemcells.2007-0197

Chua, L. S. (2014). Review on liver inflammation and antiinflammatory activity of Andrographis paniculata for hepatoprotection.Phytotherapy Research : PTR , 28 (11), 1589-1598. https://doi.org/10.1002/ptr.5193

de Crombrugghe, B., Lefebvre, V., Behringer, R. R., Bi, W., Murakami, S., \& Huang, W. (2000). Transcriptional mechanisms of chondrocyte differentiation. Matrix Biology , 19 (5), 389-394. https://doi.org/https://doi.org/10.1016/S0945-053X(00)00094-9

Fan, Z., Chubinskaya, S., Rueger, D. C., Bau, B., Haag, J., \& Aigner, T. (2004). Regulation of anabolic and catabolic gene expression in normal and osteoarthritic adult human articular chondrocytes by osteogenic protein-1. Clinical and Experimental Rheumatology ,22 (1), 103-106.

Gerwin, N., Hops, C., \& Lucke, A. (2006). Intraarticular drug delivery in osteoarthritis. Advanced Drug Delivery Reviews , 58 (2), 226-242. https://doi.org/10.1016/j.addr.2006.01.018 
Haringman, J. J., Ludikhuize, J., \& Tak, P. P. (2004). Chemokines in joint disease: the key to inflammation? Annals of the Rheumatic Diseases , 63 (10), 1186-1194. https://doi.org/10.1136/ard.2004.020529

Jensen, T., Baas, J., Dolathshahi-Pirouz, A., Jacobsen, T., Singh, G., Nygaard, J. V, .. Søballe, K. (2011). Osteopontin functionalization of hydroxyapatite nanoparticles in a PDLLA matrix promotes bone formation. Journal of Biomedical Materials Research Part A , 99A (1), 94-101. https://doi.org/10.1002/jbm.a.33166

Katagiri, K., Matsukura, Y., Muneta, T., Ozeki, N., Mizuno, M., Katano, H., \& Sekiya, I. (2017). Fibrous Synovium Releases Higher Numbers of Mesenchymal Stem Cells Than Adipose Synovium in a Suspended Synovium Culture Model. Arthroscopy - Journal of Arthroscopic and Related Surgery , 33 (4), 800-810. https://doi.org/10.1016/j.arthro.2016.09.033

Kean, W. F., Kean, R., \& Buchanan, W. W. (2004). Osteoarthritis: Symptoms, signs and source of pain. Inflammopharmacology ,12 (1), 3-31. https://doi.org/10.1163/156856004773121347

Khan, I., Khan, F., Farooqui, A., \& Ansari, I. A. (2018). Andrographolide Exhibits Anticancer Potential Against Human Colon Cancer Cells by Inducing Cell Cycle Arrest and Programmed Cell Death via Augmentation of Intracellular Reactive Oxygen Species Level.Nutrition and Cancer , 70 (5), 787-803. https://doi.org/10.1080/01635581.2018.1470649

Lajeunesse, D. (2004). The role of bone in the treatment of osteoarthritis11Supported by Procter \& Gamble Pharmaceuticals, Mason, OH. Osteoarthritis and Cartilage , 12 , 34-38. https://doi.org/https://doi.org/10.1016/j.joca.2003.09.013

Lawrence, R. C., Felson, D. T., Helmick, C. G., Arnold, L. M., Choi, H., Deyo, R. A., .. Wolfe, F. (2008). Estimates of the prevalence of arthritis and other rheumatic conditions in the United States. Part II. Arthritis and Rheumatism , 58 (1), 26-35. https://doi.org/10.1002/art.23176

Lee, K.-S., Kim, H.-J., Li, Q.-L., Chi, X.-Z., Ueta, C., Komori, T., ... Bae, S.-C. (2000). Runx2 Is a Common Target of Transforming Growth Factor $\beta 1$ and Bone Morphogenetic Protein 2, and Cooperation between Runx2 and Smad5 Induces Osteoblast-Specific Gene Expression in the Pluripotent Mesenchymal Precursor Cell Line C2C12. Molecular and Cellular Biology , 20 (23), 8783 LP - 8792. https://doi.org/10.1128/MCB.20.23.8783-8792.2000

Liao, H.-C., Chou, Y.-J., Lin, C.-C., Liu, S.-H., Oswita, A., Huang, Y.-L., . . Fu, S.-L. (2019). Andrographolide and its potent derivative exhibit anticancer effects against imatinib-resistant chronic myeloid leukemia cells by downregulating the Bcr-Abl oncoprotein.Biochemical Pharmacology , 163 , 308-320. https://doi.org/10.1016/j.bcp.2019.02.028

Lindfors, N. C., Heikkilä, J. T., \& Aho, A. J. (2008). Long-term evaluation of blood silicon and ostecalcin in operatively treated patients with benign bone tumors using bioactive glass and autogenous bone. Journal of Biomedical Materials Research Part B: Applied Biomaterials , 87B (1), 73-76. https://doi.org/10.1002/jbm.b.31070

Long, M. W. (2001). Osteogenesis and Bone-Marrow-Derived Cells.Blood Cells, Molecules, and Diseases , 27 (3), 677-690. https://doi.org/https://doi.org/10.1006/bcmd.2001.0431

Lu, W. J., Lin, K. H., Hsu, M. J., Chou, D. S., Hsiao, G., \& Sheu, J. R. (2012). Suppression of NF-kappaB signaling by andrographolide with a novel mechanism in human platelets: regulatory roles of the p38 MAPK-hydroxyl radical-ERK2 cascade. Biochemical Pharmacology ,84 (7), 914-924. https://doi.org/10.1016/j.bcp.2012.06.030

Matsumoto, T., Gargosky, S. E., Iwasaki, K., \& Rosenfeld, R. G. (1996). Identification and characterization of insulin-like growth factors (IGFs), IGF-binding proteins (IGFBPs), and IGFBP proteases in human synovial fluid. The Journal of Clinical Endocrinology and Metabolism , 81 (1), 150-155. https://doi.org/10.1210/jcem.81.1.8550744 
Mussbacher, M., Salzmann, M., Brostjan, C., Hoesel, B., Schoergenhofer, C., Datler, H., ... Schmid, J. A. (2019). Cell Type-Specific Roles of NF-kappaB Linking Inflammation and Thrombosis. Frontiers in Immunology , 10 , 85. https://doi.org/10.3389/fimmu.2019.00085

Paemanee, A., Hitakarun, A., Wintachai, P., Roytrakul, S., \& Smith, D. R. (2019). A proteomic analysis of the anti-dengue virus activity of andrographolide. Biomedicine $\&$ Pharmacotherapy $=$ Biomedecine $\&$ Pharmacotherapie , 109 , 322-332. https://doi.org/10.1016/j.biopha.2018.10.054

Pholphana, N., Rangkadilok, N., Thongnest, S., Ruchirawat, S., Ruchirawat, M., \& Satayavivad, J. (2004). Determination and variation of three active diterpenoids in Andrographis paniculata (Burm.f.) Nees.Phytochemical Analysis , 15 (6), 365-371. https://doi.org/10.1002/pca.789

Sandell, L. J., \& Aigner, T. (2001). Articular cartilage and changes in arthritis. An introduction: cell biology of osteoarthritis.Arthritis Research , 3 (2), 107-113. https://doi.org/10.1186/ar148

Shaughnessy, S., Smith, E. R., Kodukula, S., Storch, J., \& Fried, S. K. (2000). Adipocyte metabolism in adipocyte fatty acid binding protein knockout mice (aP2-/-) after short-term high-fat feeding: functional compensation by the keratinocyte [correction of keritinocyte] fatty acid binding protein. Diabetes , 49 (6), 904-911. https://doi.org/10.2337/diabetes.49.6.904

Singha, P. K., Roy, S., \& Dey, S. (2007). Protective activity of andrographolide and arabinogalactan proteins from Andrographis paniculata Nees. against ethanol-induced toxicity in mice. Journal of Ethnopharmacology , 111 (1), 13-21. https://doi.org/10.1016/j.jep.2006.10.026

Steinberg, J., \& Zeggini, E. (2016). Functional genomics in osteoarthritis: Past, present, and future. Journal of Orthopaedic Research : Official Publication of the Orthopaedic Research Society ,34 (7), 1105-1110. https://doi.org/10.1002/jor.23296

Sweeney, S. E., \& Firestein, G. S. (2004). Signal transduction in rheumatoid arthritis. Current Opinion in Rheumatology ,16 (3), 231-237. https://doi.org/10.1097/00002281-200405000-00011

Tan, W. S. D., Liao, W., Zhou, S., \& Wong, W. S. F. (2017). Is there a future for andrographolide to be an anti-inflammatory drug? Deciphering its major mechanisms of action. Biochemical Pharmacology ,139, 71-81. https://doi.org/10.1016/j.bcp.2017.03.024

Wintachai, P., Kaur, P., Lee, R. C. H., Ramphan, S., Kuadkitkan, A., Wikan, N., ... Smith, D. R. (2015). Activity of andrographolide against chikungunya virus infection. Scientific Reports ,5 , 14179. https://doi.org/10.1038/srep14179

Xu, H. E., Lambert, M. H., Montana, V. G., Parks, D. J., Blanchard, S. G., Brown, P. J., ... Milburn, M. V. (1999). Molecular Recognition of Fatty Acids by Peroxisome Proliferator-Activated Receptors. Molecular Cell , 3 (3), 397-403. https://doi.org/https://doi.org/10.1016/S1097-2765(00)80467-0

Zhai, Z. J., Li, H. W., Liu, G. W., Qu, X. H., Tian, B., Yan, W., .. Dai, K. R. (2014). Andrographolide suppresses RANKL-induced osteoclastogenesis in vitro and prevents inflammatory bone loss in vivo. British Journal of Pharmacology , 171 (3), 663-675. https://doi.org/10.1111/bph.12463

\section{Hosted file}

Figure and Table - final.docx available at https://authorea.com/users/339040/articles/465336novel-dose-dependent-effects-of-andrographolide-on-the-enhancement-of-chondrogenesisand-osteogenesis-in-human-mesenchymal-stem-cells

\section{Hosted file}

Picture final.docx available at https://authorea.com/users/339040/articles/465336-noveldose-dependent-effects-of-andrographolide-on-the-enhancement-of-chondrogenesis-andosteogenesis-in-human-mesenchymal-stem-cells 